\title{
Centrifugally stimulated exospheric ion escape at Mercury
}

\author{
D. C. Delcourt, ${ }^{1}$ K. Seki, ${ }^{2}$ N. Terada, ${ }^{3}$ and T. E. Moore ${ }^{4}$ \\ Received 1 October 2012; revised 26 October 2012; accepted 26 October 2012; published 30 November 2012.
}

[1] We investigate the transport of ions in the low-altitude magnetosphere of Mercury. We show that, because of small spatial scales, the centrifugal effect due to curvature of the $\mathbf{E} \times \mathbf{B}$ drift paths can lead to significant particle energization in the parallel direction. We demonstrate that because of this effect, ions with initial speed smaller than the escape speed such as those produced via thermal desorption can overcome gravity and escape into the magnetosphere. The escape route of this low-energy exosphere originating material is largely controlled by the magnetospheric convection rate. This escape route spreads over a narrower range of altitudes when the convection rate increases. Bulk transport of low-energy planetary material thus occurs within a limited region of space once moderate magnetospheric convection is established. These results suggest that, via release of material otherwise gravitationally trapped, the E $\times \mathbf{B}$ related centrifugal acceleration is an important mechanism for the net supply of plasma to the magnetosphere of Mercury. Citation: Delcourt, D. C., K. Seki, N. Terada, and T. E. Moore (2012), Centrifugally stimulated exospheric ion escape at Mercury, Geophys. Res. Lett., 39, L22105, doi:10.1029/2012GL054085.

\section{Introduction}

[2] Following the pioneering observations of Mariner-10 in 1974-75, in-situ measurements from the MESSENGER spacecraft confirm the existence of an intrinsic magnetic field at Mercury [e.g., Anderson et al., 2011] ; hence, the formation of a miniature magnetosphere with spatial and temporal scales much smaller than those at Earth (by about a factor 8 and a factor 40, respectively). The typical standoff distance of Mercury's magnetopause is expected to be of the order of 1.5-2 $R_{M}$ [e.g., Slavin et al., 2010a, 2010b]. Material of planetary origin can be released into the exosphere of Mercury via a variety of processes such as thermal desorption, electron-stimulated desorption [e.g., McLain et al., 2011], photon-stimulated desorption, solar wind sputtering or micro-meteoritic bombardment [e.g., Leblanc and Johnson, 2003]. Once ionized by solar UVs, these newly formed ions of planetary origin are subjected to a large-scale transport and contribute to the different regions of Mercury's magnetosphere, as found from MESSENGER observations and simulation modeling [e.g., Zurbuchen et al.,

\footnotetext{
${ }^{1}$ LPP, CNRS, Saint-Maur des Fossés, France.

${ }^{2}$ STELAB, Nagoya University, Nagoya, Japan.

${ }^{3}$ Department of Geophysics, Tohoku University, Sendai, Japan.

${ }^{4}$ NASA Goddard Space Flight Center, Greenbelt, Maryland, USA.
}

Corresponding author: D. C. Delcourt, LPP, CNRS, 4, avenue de Neptune, FR-94107 Saint-Maur des Fossés CEDEX, France. (dominique.delcourt@lpp.polytechnique.fr)

(C)2012. American Geophysical Union. All Rights Reserved. 0094-8276/12/2012GL054085
2008, 2011; Delcourt et al., 2003; Paral et al., 2010]. The purpose of this study is to examine the impact of centrifugal effects in the miniature environment of Mercury on the fate of exospheric material. These centrifugal effects will first be described in section 2. In section 3 , we will examine their consequences on the transport of low-energy ions.

\section{Centrifugal Acceleration}

[3] In the adiabatic (guiding center) approximation, the parallel equation of motion in the absence of parallel electric field writes [see, e.g., Northrop, 1963, equation (1.20)]:

$$
\dot{V}_{\|}=\mathrm{g}_{\|}+\mathbf{V}_{E} \cdot\left[\frac{\partial \mathbf{b}}{\partial t}+V_{\|} \frac{\partial \mathbf{b}}{\partial s}+\mathbf{V}_{E} \cdot \nabla \mathbf{b}\right]-\frac{\mu}{m} \frac{\partial B}{\partial s}
$$

Here, $\mathrm{g}_{\|}$is the gravitational acceleration in the parallel direction, $\mathbf{V}_{E}$ is the $\mathbf{E} \times \mathbf{B}$ drift velocity, $V_{\|}$, the particle parallel speed, $\mathbf{b}$, a unit vector in the $\mathbf{B}$ direction, $s$, the curvilinear abscissa along the field line, and $m$ and $\mu$, the particle mass and magnetic moment, respectively. Equation (1) displays two distinct curvature related acceleration terms. The third term on the right hand side of (1) is due to curvature of the magnetic field lines and can lead to quite substantial acceleration upon crossing of a field reversal. The fourth term on the right hand side of (1) is due to curvature of the $\mathbf{E} \times \mathbf{B}$ drift paths, a centrifugal acceleration that can be viewed as that affecting a bead (the charged particle) on a rotating rod (the convecting magnetic field line) [e.g., Horwitz et al., 1994]. In the Earth's magnetosphere, it was shown that this latter $\mathbf{E} \times \mathbf{B}$ related centrifugal effect can lead to upward ion energization of a few eVs or a few tens of eVs at low altitudes [e.g., Cladis, 1986] or to focusing (referred to as "centrifugal trapping") of slow plasma sheet particles into the equatorial region [e.g., Delcourt et al., 1990]. Because of small spatial scales, it was shown by Delcourt et al. [2002] that this $\mathbf{E} \times \mathbf{B}$ related centrifugal acceleration is more pronounced at Mercury than at Earth, possibly yielding energization of planetary material up to several hundreds of $\mathrm{eV}$ within minutes when ions are transported into the magnetospheric lobes over the polar cap [see, e.g., Delcourt et al., 2002, Figure 1]. Assuming a steady magnetic field configuration, this acceleration occurs in an adiabatic (magnetic moment conserving) manner since, in this region of space, the magnetic field does not significantly change within a cyclotron turn. As shown in Delcourt et al. [1990, section 3.2], an equation similar to (1) can be obtained for the full particle with the exception of the mirror force term (last term in (1)). The centrifugal acceleration described above thus does not depend upon the adiabatic or nonadiabatic character of the motion.

[4] The centrifugal acceleration due to curvature of the $\mathbf{E} \times \mathbf{B}$ drift paths is the one of interest in this study since it opposes the downward oriented gravitational acceleration 

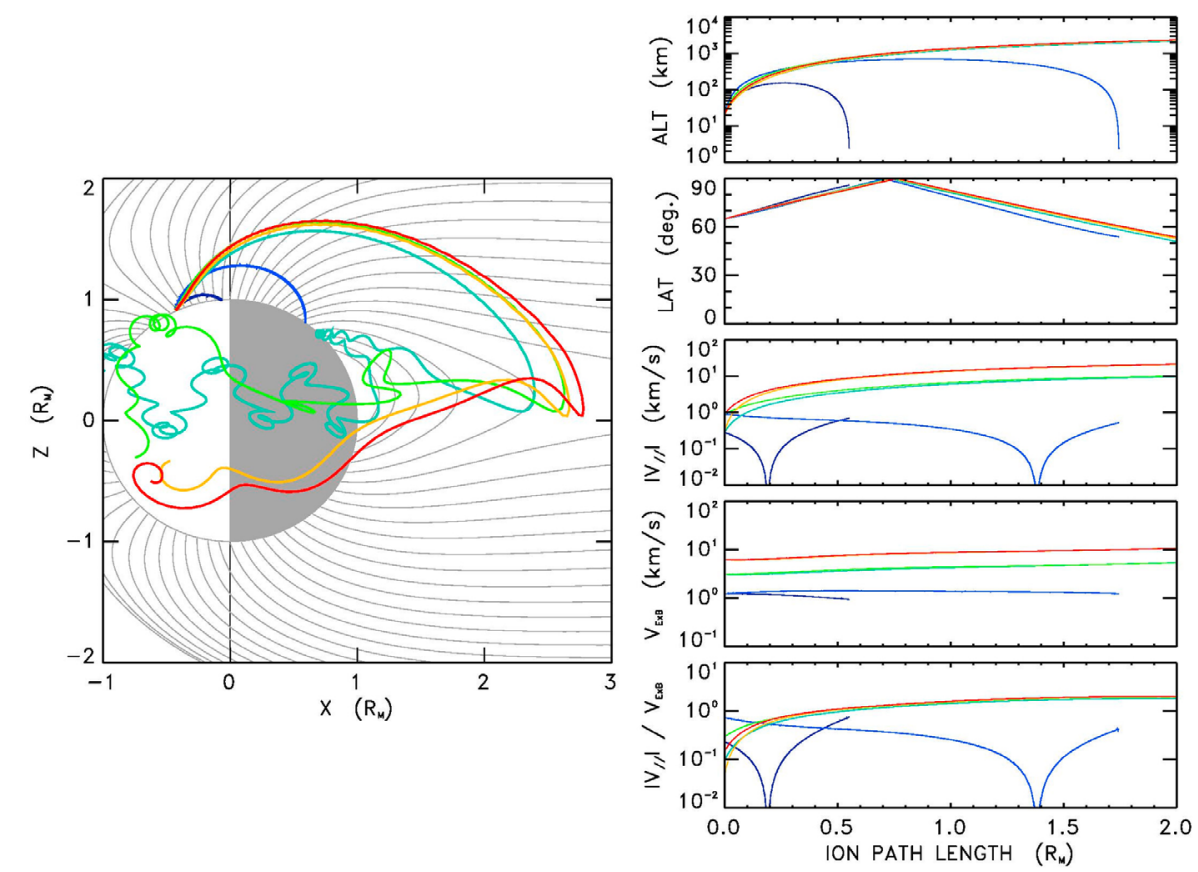

Figure 1. (left) $\mathrm{Na}^{+}$trajectories in the $X-Z$ plane. (right) From top to bottom, $\mathrm{Na}^{+}$altitude, latitude, parallel speed, $\mathbf{E} \times \mathbf{B}$ drit speed, and parallel speed-to-E $\times \mathbf{B}$ drift speed ratio as a function of path length. The test ions are launched from the planet surface at $65^{\circ}$ latitude in the dayside sector, with $0.01 \mathrm{eV}$ or $0.1 \mathrm{eV}$ energy, $170^{\circ}$ pitch angle and arbitrary gyrophase. Blue, green and orange-red colors correspond to cross-polar cap potential drops of $2 \mathrm{kV}, 5 \mathrm{kV}$, and $10 \mathrm{kV}$, respectively.

$\left(g_{\|}\right.$in (1)). It can thus affect the net upward transport of ions depending upon magnitude of the magnetospheric convection. To quantitatively investigate this effect with the help of numerical simulations, we adopt as in previous studies [e.g., Delcourt et al., 2003] the model of Luhmann and Friesen [1979] that consists of the superposition of a dipole field with a Harris sheet. As suggested by recent MESSENGER measurements [Anderson et al., 2011], we also take into account a northward offset of $484 \mathrm{~km}$ for the magnetic field, and we use the following model parameters : $B_{T}=70 \mathrm{nT}$, $L=0.5 R_{M}$, and $B_{E Q}=195 \mathrm{nT}\left(B_{T}\right.$ being the asymptotic tail field, $L$, the half-thickness of the current sheet and $B_{E Q}$, the equatorial surface field). As for the large-scale convection electric field, we use in first approximation a two-cell VollandStern pattern [e.g., Volland, 1978] that leads to antisunward transport in the polar cap region and sunward transport below. Distinct cross-polar cap potential drops are considered as described hereinafter. As for the test particle trajectories, they were computed using the full equation of motion, integrated by means of a fourth order Runge-Kutta technique.

[5] Figure 1 shows an example of the ion trajectories obtained. We consider here the sodium population that is released into Mercury's exosphere at very low energies (less than $0.1 \mathrm{eV}$ ) as expected for instance from thermal desorption [see, e.g., Leblanc and Johnson, 2003, Figure 3]. Once ionized by solar UVs, the newly produced $\mathrm{Na}^{+}$ions are entrained by the convecting magnetic field lines and circulate inside the magnetosphere. The trajectories coded in blue in Figure 1 (left) show the results obtained for $0.01 \mathrm{eV}$ and $0.1 \mathrm{eV} \mathrm{Na}^{+}$originating from the planet vicinity $(5 \mathrm{~km}$ altitude) in the high-latitude dayside sector. In this case, a weak convection electric field is considered, with a cross-polar potential drop of $2 \mathrm{kV}$. It can be seen in this panel that, due to initial speeds smaller than the escape speed, the ions are trapped by the gravitational field, viz., they travel upward until some maximum altitude at which point they are turned back toward the planet and subsequently precipitate onto the surface (note here that during this transport, the test $\mathrm{Na}^{+}$with $0.1 \mathrm{eV}$ initial energy is convected into the nightside sector over the polar cap). This gravitationally bound behavior can be better appreciated in Figure 1 (right) that show the ion position and velocity as a function of path length. It can be seen that the $\mathrm{Na}^{+}$with $0.01 \mathrm{eV}$ and $0.1 \mathrm{eV}$ energy reach maximum altitudes of $\sim 150 \mathrm{~km}$ and $\sim 700 \mathrm{~km}$, respectively. As expected, their parallel velocity (third panel from top) first decreases, then increases upon return toward the planet.

[6] The trajectories coded in green and orange-red in Figure 1 were obtained considering larger cross-polar cap potential drops (viz., $5 \mathrm{kV}$ and $10 \mathrm{kV}$, respectively). In both cases, a behavior radically different than trapping at low altitudes (blue trajectories) can be seen. That is, regardless of initial energy, the test $\mathrm{Na}^{+}$are now transported over the polar cap into the nightside sector where they eventually interact with the magnetotail current sheet. Not surprisingly, during the lobe trajectory sequence, the adiabaticity parameter $\kappa$ that is defined as the square root of the minimum curvature radius-to-maximum Larmor radius ratio [e.g., Büchner and Zelenyi, 1989] is larger than 10 (its initial value being $\geq 90$ ) and, in this region of space, the ion motion is thus adiabatic (magnetic moment conserving). In contrast, during interaction with the magnetotail current sheet, the ions are subjected to large magnetic moment change and nonadiabatic energization (of several keVs). These ions substantially contribute to the plasma sheet populations prior to traveling back toward the planet and precipitating in the dusk sector. Figure 1 (left) shows that the green and orangered trajectories intercept the pole at altitudes significantly higher than the blue ones. As demonstrated by Delcourt et al. 

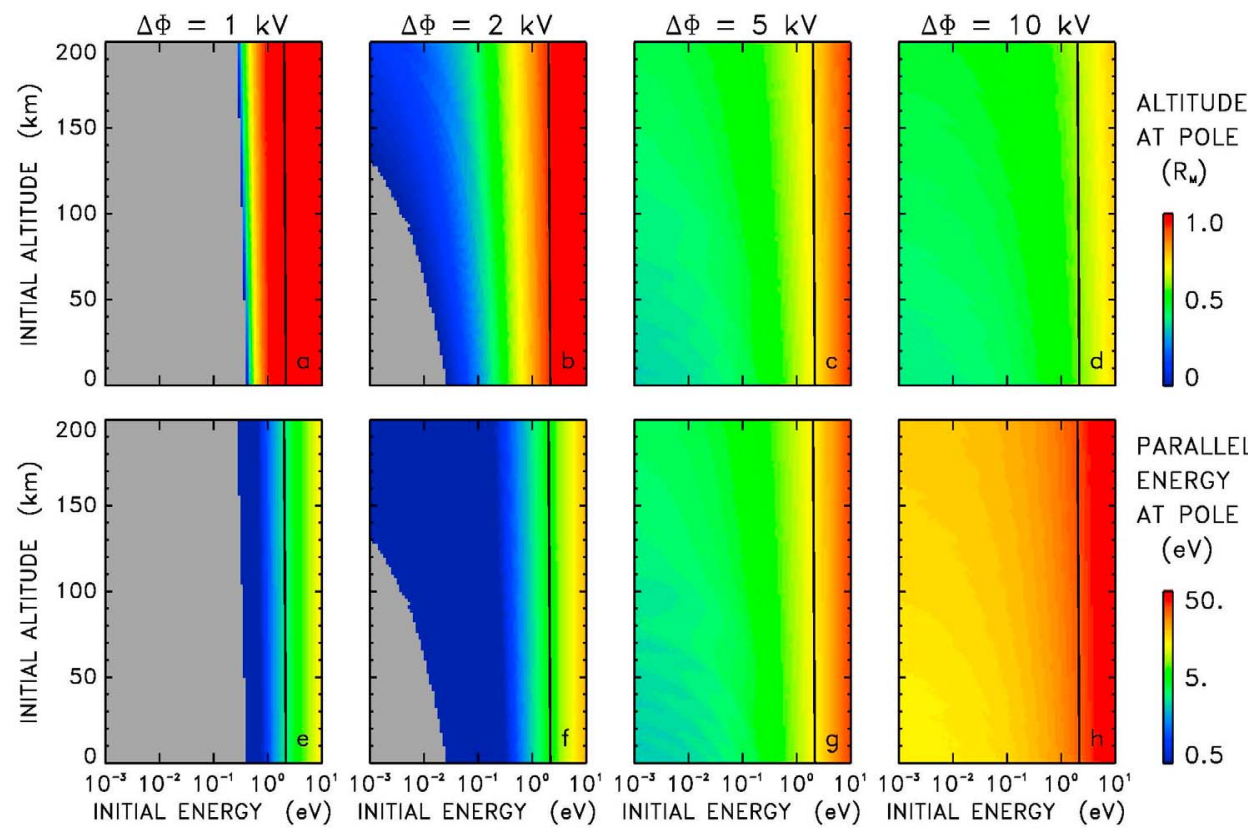

Figure 2. Color-coded altitude (a-d) and parallel energy (e-h) of $\mathrm{Na}^{+}$ions at entry into the nightside sector as a function of initial energy and altitude. The test ions are launched from the dayside sector at $65^{\circ}$ latitude with $170^{\circ}$ pitch angle and arbitrary gyrophase. Different cross-polar cap potential drop are considered from left to right: $1 \mathrm{kV}, 2 \mathrm{kV}, 5 \mathrm{kV}$, and $10 \mathrm{kV}$. The grey shaded area corresponds to ions that do not cross the pole.

[2002], the reason for this is that their parallel speed rapidly increases after ejection into the magnetosphere under the effect of a large $\mathbf{E} \times \mathbf{B}$ related centrifugal effect. Upon crossing of the pole, their altitudes are of the order of $0.5 \mathrm{R}_{M}$. Clearly, the low-energy $\mathrm{Na}^{+}$now rapidly overcome the gravitational field and can travel up to large distances in the Hermean magnetosphere.

[7] It is also of particular interest to note in Figure 1 that, although two distinct cross-polar cap potential drops are considered for the trajectories coded in green and orangered, the altitudes at pole crossing as well as the trajectory apex in the nightside sector are fairly similar. Looking at Figure 1 (right), it can be seen that the $\mathbf{E} \times \mathbf{B}$ drift speed along the orange-red trajectories is twice larger than that along the green ones, which should thus lead to a different drift path. However, the parallel speed obtained from $\mathbf{E} \times \mathbf{B}$ related centrifugal acceleration also increases in the same proportion (Figure 1, bottom right) so that the net ion drift path is not much affected. In other words, Figure 1 demonstrates that the $\mathbf{E} \times \mathbf{B}$ related centrifugal acceleration can be significant enough for ions to overcome gravitational trapping and to escape into the Hermean magnetosphere. Moreover, their escape route appears to depend little upon $\mathbf{E} \times \mathbf{B}$ magnitude since the ion parallel speed increases in proportion to it. Given the large amount of planetary material that is expelled at low energies from the planet surface, this centrifugal escape mechanism may play a significant role in the net supply of plasma to the magnetosphere.

\section{Centrifugal Escape}

[8] To better characterize the above centrifugal escape mechanism, we performed systematic trajectory computations for $\mathrm{Na}^{+}$with different initial energies $(0.001 \mathrm{eV}-$ $10 \mathrm{eV}$ ) and initial altitudes (below $200 \mathrm{~km}$ ). The results of these computations are presented in Figure 2. Here, to explore the large variety of ion behaviors (from gravitationally bound, as with the blue trajectories in Figure 1, to mostly bunched together during escape, as with the orangered trajectories), we consider a wide range of cross-polar cap potential drops, from extremely weak $(1 \mathrm{kV})$ to fairly large values $(10 \mathrm{kV})$. This leads to a dawn-to-dusk convection electric field in the equatorial magnetotail that varies between $\sim 0.3 \mathrm{mV} . \mathrm{m}^{-1}$ and $\sim 3.0 \mathrm{mV} \cdot \mathrm{m}^{-1}$. The test $\mathrm{Na}^{+}$in Figure 2 are launched from $65^{\circ}$ latitude in the dayside sector on the noon meridian. For those ions that escape into the magnetosphere, Figures 2 (top) and 2 (bottom) show the color-coded altitude and parallel energy at pole crossing or, equivalently, upon entry into the nightside sector. It can be seen in Figures 2a and 2e that, for very weak magnetospheric convection, most of the test $\mathrm{Na}^{+}$considered return to the planet before crossing the pole. As expected, only those ions with initial speed comparable to or larger than the escape speed (corresponding to an initial energy of $\sim 2 \mathrm{eV}$ as indicated by the solid black line) gain access to the nightside sector.

[9] On the other hand, if a larger convection rate is considered (from left to right in Figure 2), the amount of ions that enter the nightside magnetosphere significantly increases. As a matter of fact, for potential drops of $5 \mathrm{kV}$ and above, all test ions in the initial altitude and energy ranges considered escape into the magnetosphere (Figure 2). In particular, the comparison of Figures $2 \mathrm{~g}$ and $2 \mathrm{~h}$ reveals that the $\mathrm{Na}^{+}$parallel energy at pole crossing increases when the convection rate increases, as expected from a more pronounced $\mathbf{E} \times \mathbf{B}$ related centrifugal acceleration. However, as discussed in Figure 1, this enhanced parallel acceleration does not translate as widely different altitudes upon crossing of the pole. As a matter of fact, comparison of Figures $2 \mathrm{c}$ 

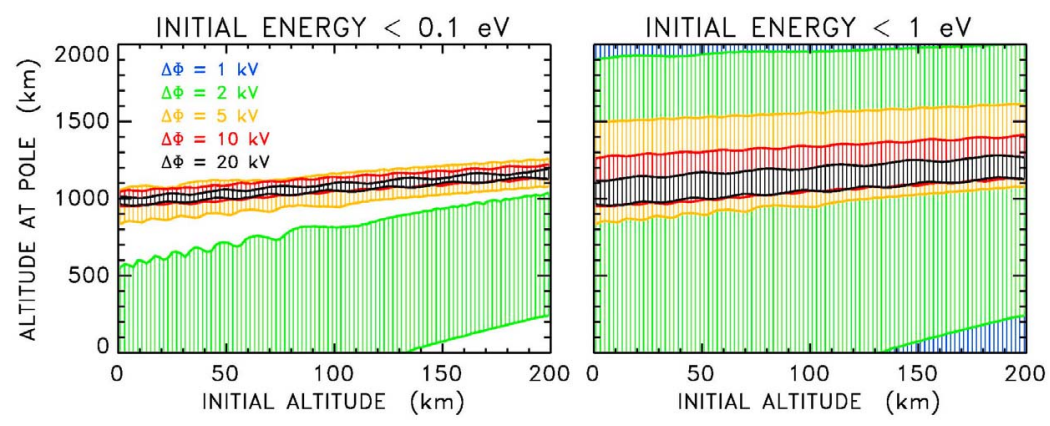

Figure 3. Envelope of $\mathrm{Na}^{+}$altitudes at entry into the nightside sector as a function of initial altitude and for different (colorcoded) cross-polar cap potential drops. Ions with initial energy smaller than (left) $0.1 \mathrm{eV}$ and (right) $1 \mathrm{eV}$.

and $2 \mathrm{~d}$ shows that, as the convection electric field increases, the range of altitudes at pole crossing gradually decreases. This suggests that, as a result of $\mathbf{E} \times \mathbf{B}$ related centrifugal focusing, there exists some limiting trajectory toward which the drift paths of all low-energy ions converge.

[10] This effect can be better appreciated in Figure 3 that shows the envelope of $\mathrm{Na}^{+}$altitudes at pole crossing as a function of initial altitude and for all energies below $0.1 \mathrm{eV}$ (Figure 3, left) and below $1 \mathrm{eV}$ (Figure 3, right). The different colors in this figure correspond to distinct convection rates. It can be seen in Figure 3 that, as the cross-polar cap potential drop increases, the range of altitudes at which the low-energy ions enter the nightside sector significantly shrinks until some narrow channel is obtained (black lines in Figure 3). In other words, the pattern in black in Figure 3 delineates some asymptotic regime in which all low-energy ions released at low altitudes overcome gravity and travel into the nightside magnetosphere.

[11] The pattern achieved in Figure 3 was obtained for an initial latitude of $65^{\circ}$. For different initial latitudes, patterns similar to that in Figure 3 are obtained but at different altitudes. Accordingly, it can be speculated that the outermost altitudes at which low-energy ions originating from the dayside enter the nightside sector is associated with a notable change of density, a feature reminiscent of the "geopause" suggested by Moore and Delcourt [1995] to delineate the domain of planetary plasma contribution in the terrestrial magnetosphere. This can be better appreciated in Figure 4 that presents the results of systematic trajectory calculations, considering different initial latitudes in the dayside sector. Here, a number of test $\mathrm{Na}^{+}$were launched in the 0-200 km altitudinal range (by steps of $1 \mathrm{~km}$ ) above $50^{\circ}$ latitude (by steps of $1^{\circ}$ ) in the dayside sector, with distinct pitch angles and energies (less than $0.1 \mathrm{eV}$ ). Once the trajectory calculations were performed, the density and average energy in a given altitudinal bin were calculated by summation over the various $\mathrm{Na}^{+}$occurring in that bin, in a like manner to Delcourt et al. [2003] (see equations (2)-(3) of that paper). In Figure 4 (left) that shows the ion altitude at pole crossing versus initial latitude, it is clearly apparent that the larger the convection rate, the smaller the altitudinal spread for given initial latitude. In other words, enhanced $\mathbf{E} \times \mathbf{B}$ related centrifugal acceleration yields enhanced structuring of the transpolar plasma flow. In terms of density, it is apparent from Figure 4 (middle) that this translates as a more abrupt variation at high altitude. That is, whereas the ion density gradually decreases with altitude for weak convection (blue profile), a more "step-like" behavior is obtained for enhanced convection (red profile). Finally, as expected, Figure 4 (right) displays significantly larger total energy (solid lines) and parallel energy (dotted lines) at entry into the nightside sector when the convection rate increases.

[12] It was mentioned above that the MESSENGER measurements provide evidence of a northward offset of $484 \mathrm{~km}$ of the magnetic field [Anderson et al., 2011]. This offset that was taken into account in the present modeling
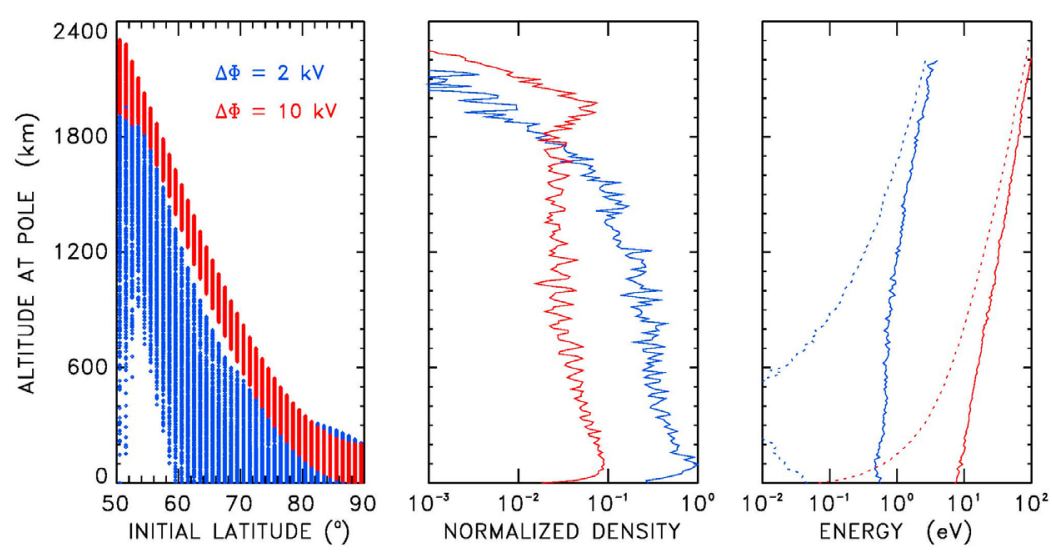

Figure 4. (left) Initial latitude, (middle) density and (right) energy of $\mathrm{Na}^{+}$ions versus altitude at pole crossing. Blue and red profiles correspond to two different cross-polar cap potential drops ( $2 \mathrm{kV}$ and $10 \mathrm{kV}$, respectively). The dotted lines in Figure (right) show the $\mathrm{Na}^{+}$parallel energy. 
(see section 2), leads to a stronger surface magnetic field at high northern latitudes as compared to southern ones. With a larger polar cap in the southern hemisphere as compared to the northern one, this offset may affect the ejection of exospheric material in each hemisphere. It should also lead to some asymmetry in the ion flows in northern and southern lobes. As for the centrifugal escape mechanism, it depends upon the magnitude of both convection electric field and curvature of the $\mathbf{E} \times \mathbf{B}$ drift paths. This mechanism should thus operate in both hemispheres, albeit with different efficiencies (i.e., different limiting energies for bulk escape) at northern and southern latitudes. This efficiency variation is clearly reflected in Figure 2, with a change in the amount of escaping ions from $\sim 35 \%$ up to $100 \%$ of the total population considered as the convection rate increases. Centrifugally stimulated escape may explain the presence of $\mathrm{Ca}^{+}$ions in the near-Mercury magnetotail during M3 [Vervack et al., 2010], a MESSENGER flyby that was characterized by intense magnetospheric convection. A thorough assessment of this centrifugal mechanism efficiency awaits additional in-situ measurements for a variety of magnetospheric conditions. The MESSENGER measurements also provide numerous observations of Flux Transfer Events (FTE) at Mercury [e.g., Slavin et al., 2010a, 2010b]. At Earth, such localized reconnection phenomena go together with narrow channels of enhanced convection at low altitudes [e.g., Pinnock et al., 1993]. If this is also the case at Mercury, in view of the present results, such FTEs may lead to $\mathbf{E} \times \mathbf{B}$ related centrifugal escape of otherwise trapped particles, hence ripping off low-energy exospheric material and releasing it into interplanetary space.

\section{Conclusion}

[13] Because of the small spatial scales of Mercury's magnetosphere, the centrifugal acceleration due to curvature of the $\mathbf{E} \times \mathbf{B}$ drift paths can lead to significant ion energization in the parallel direction. As a result, large amounts of low-energy material in the immediate vicinity of the planet can overcome gravitational trapping and escape into the magnetosphere. The larger the convection rate, the more efficient the centrifugal focusing of these escaping ions during transport from dayside to nightside, the larger the limiting energy below which bulk transport is obtained. This $\mathbf{E} \times \mathbf{B}$ related centrifugal mechanism may play a prominent role in the net plasma supply to the Hermean magnetosphere and/or in the escape of planetary material into interplanetary space.
[14] Acknowledgments. The Editor thanks two anonymous reviewers for their assistance in evaluating this paper.

\section{References}

Anderson, B. J., et al. (2011), The global magnetic field of Mercury from MESSENGER orbital observations, Science, 333, 1859, doi:10.1126/ science. 1211001

Büchner, J., and L. M. Zelenyi (1989), Regular and chaotic charged particle motion in magnetotaillike field reversals: 1. Basic theory of trapped motion, J. Geophys. Res., 94, 11,821, doi:10.1029/JA094iA09p11821.

Cladis, J. B. (1986), Parallel acceleration and transport of ions from polar ionosphere to plasma sheet, Geophys. Res. Lett., 13, 893, doi:10.1029/ GL013i009p00893.

Delcourt, D. C., J. A. Sauvaud, and A. Pedersen (1990), Dynamics of single-particle orbits during substorm expansion phase, J. Geophys. Res., 95, 20,853, doi:10.1029/JA095iA12p20853.

Delcourt, D. C., T. E. Moore, S. Orsini, A. Millilo, and J.-A. Sauvaud (2002), Centrifugal acceleration of ions near Mercury, Geophys. Res. Lett., 29(12), 1591, doi:10.1029/2001GL013829.

Delcourt, D. C., et al. (2003), A quantitative model of planetary $\mathrm{Na}^{+}$contribution to Mercury's magnetosphere, Ann. Geophys., 21, 1723 doi:10.5194/angeo-21-1723-2003.

Horwitz, J. L., C. W. Ho, H. D. Scarbro, G. R. Wilson, and T. E. Moore (1994), Centrifugal acceleration of the polar wind, J. Geophys. Res., 99, 15,051, doi:10.1029/94JA00924.

Leblanc, F., and R. E. Johnson (2003), Mercury's sodium exosphere, Icarus, 164, 261, doi:10.1016/S0019-1035(03)00147-7.

Luhmann, J. G., and L. M. Friesen (1979), A simple model of the magnetosphere, J. Geophys. Res., 84, 4405, doi:10.1029/JA084iA08p04405.

McLain, J. L., A. L. Sprague, G. A. Grieves, D. Schriver, P. Travinicek, and T. M. Orlando (2011), Electron-stimulated desorption of silicates: A potential source for ions in Mercury's space environment, J. Geophys. Res., 116, E03007, doi:10.1029/2010JE003714.

Moore, T. E., and D. C. Delcourt (1995), The geopause, Rev. Geophys., 33, 175, doi:10.1029/95RG00872.

Northrop, T. G. (1963), The Adiabatic Motion of Charged Particles, Wiley Intersci., New York.

Paral, J., P. M. Trávníček, R. Rankin, and D. Schriver (2010), Sodium ion exosphere of Mercury during MESSENGER flybys, Geophys. Res. Lett., 37, L19102, doi:10.1029/2010GL044413.

Pinnock, M., A. S. Rodger, J. R. Dudeney, K. B. Baker, P. T. Newell, R. A. Greenwald, and M. E. Greenspan (1993), Observations of an enhanced convection channel in the cusp ionosphere, J. Geophys. Res., 98, 3767, doi:10.1029/92JA01382.

Slavin, J. A., et al. (2010a), MESSENGER observations of large flux transfer events at Mercury, Geophys. Res. Lett., 37, L02105, doi:10.1029/ 2009 GL041485.

Slavin, J. A., et al. (2010b), MESSENGER observations of extreme loading and unloading of Mercury's magnetic tail, Science, 329, 665, doi:10.1126/science.1188067.

Vervack, R. J., Jr., et al. (2010), Mercury's complex exosphere: Results from MESSENGER third flyby, Science, 329, 672, doi:10.1126/science. 1188572.

Volland, H. (1978), A model of the magnetospheric convection electric field, J. Geophys. Res., 83, 2695, doi:10.1029/JA083iA06p02695.

Zurbuchen, T. H., et al. (2008), MESSENGER observations of the composition of Mercury's ionized exosphere and plasma environment, Science, 321, 90, doi:10.1126/science. 1159314 .

Zurbuchen, T. H., et al. (2011), MESSENGER observations of the spatial distribution of planetary ions near Mercury, Science, 333, 1862, doi:10.1126/ science.1211302. 